\title{
A DEFINITION OF THE EXTERIOR DERIVATIVE IN TERMS OF LIE DERIVATIVES
}

\author{
RICHARD S. PALAIS ${ }^{1}$
}

The notion of the Lie derivative of a tensor field with respect to a vector field, though much neglected, goes back to almost the beginnings of tensor analysis. For a classical treatment see [1, pp. 72-73]. A modern treatment with the simplifying assumption that the vector field does not vanish is given in [2, pp. 74-77], but little or nothing in the way of a coordinate free treatment has appeared in print so we give an abbreviated exposition below.

Lie derivatives. Let $\mathcal{H}$ be a $C^{\infty}$ manifold, $R$ the real number system with the usual $C^{\infty}$ structure. Let $\mathcal{O}$ be an open subset of $R \times \mathcal{X}$. Let $\phi$ be a $C^{\infty}$ map of $\mathcal{O}$ into $\mathcal{X}$. For each $p \in \mathcal{X}$ let $I^{(p)}$ $=[t \in R:(t, p) \in \mathcal{O}]$ and let $\sigma^{(p)}: I^{(p)} \rightarrow \mathcal{X}$ be defined by $\sigma^{(p)}(t)=\phi(t, p)$. For each $t \in R$ let $S_{t}=[p \in \mathcal{X}:(t, p) \in \mathcal{O}]$ and let $\phi_{t}: S_{t} \rightarrow \mathcal{X}$ be defined by $\phi_{t}(p)=\phi(t, p)$.

DEFINITION. $\phi$ is a one-parameter quasi-group of transformations of $\mathcal{X}$ if: (1) For each $p \in \mathcal{X}, I^{(p)}$ is an interval containing zero and $\phi(0, p)=p$; (2) If $(\tau, p),(t, \phi(\tau, p))$, and $(t+\tau, p)$ are in $\mathcal{O}$, then $\phi(t+\tau, p)=\phi(t, \phi(\tau, p))$. We say $\phi$ is maximal if it is not properly included in a one-parameter quasi-group of transformations of $\mathcal{X}$.

Let $\phi$ be a one-parameter quasi-group of transformations of $\mathcal{X}$. For each $p \in \mathcal{X}$ and each real-valued function $f$ of class $C^{\infty}$ on $\mathscr{X} C$ we define: $L_{p}(f)=(d / d t)_{t=0} f(\phi(t, p))$. It is easily verified that $L_{p}$ is a tangent vector at $p$ and that $L: p \rightarrow L_{p}$ is a $C^{\infty}$ vector field on $\mathcal{X}$ called the infinitesimal transformation of $\phi . L$ is said to generate $\phi$. The global theory of the integration of first order ordinary differential equations on a manifold is essentially contained in the following.

Theorem I. Every $C^{\infty}$ vector field $L$ on a $C^{\infty}$ manifold $\mathcal{X}$ generates a unique maximal one-parameter quasi-group of transformations of $\mathcal{X}$, $\phi^{(L)}$. A one-parameter quasi-group of transformations of $\mathcal{X}$, $\tilde{\phi}$, has infinitesimal generator $L$ if and only if it is included in $\phi^{(L)}$.

In the following $\mathcal{X}$ denotes a fixed $C^{\infty}$ manifold. If $p \in \mathcal{X}$ we denote by $\mathcal{X}_{p}$ the tangent space at $p$. By a tensor $A$ of contravariant rank $r$ and covariant rank $s$ at $p$ we shall mean an element of the space $\left(\otimes r \mathscr{C}_{p}\right) \otimes\left(\otimes \mathcal{P}_{p}^{*}\right)$ identified in the usual fashion as multilinear functionals on $\mathscr{X}_{p}^{*} \times \cdots \times \mathcal{X}_{p}^{*} \times \mathcal{X}_{p} \times \cdots \times \mathcal{X}_{p}$ (where there are $r \mathcal{X}_{p}^{* \prime}$ s

Received by the editors April 17, 1954.

1 National Science Foundation Fellow. 
and $s \mathcal{X}_{p}$ 's). If $\phi$ is a nonsingular $C^{\infty}$ map of $\mathcal{X}$ into itself defined at $p$, we denote by $\delta \phi$ not only its differential but also all the associated Kronecker products, mapping the various tensor spaces at $p$ isomorphically onto the corresponding tensor spaces at $\phi(p)$. We let the argument determine the particular map in question. Thus:

$$
\delta \phi(A)=\left(\delta \phi_{p} \otimes \cdots \otimes \delta \phi_{p} \otimes \delta \phi_{p}^{*-1} \otimes \cdots \otimes \delta \phi_{p}^{*-1}\right) A
$$

(where there are $r \delta \phi_{p}$ 's and $s \delta \phi_{p}^{*-1}$ 's).

In what follows let $L$ be a fixed $C^{\infty}$ vector field on $\mathcal{X}$ and $\phi$ the maximal one-parameter quasi-group it generates. If $p \in \mathcal{X}$, then for $t$ sufficiently small (e.g. contained in $I^{(p)}$ ) $\phi_{-t}$ is defined in a neighborhood of $\phi_{t}(p)$ and maps the latter point on $p$. We now can make the following definition:

Definition. Let $A$ be a tensor field on $\mathcal{X}$ and $p \in \mathcal{X}$. If $(d / d t)_{t=0} \delta \phi_{-t}\left(A_{\phi_{t}(p)}\right)$ exists we denote it by $L[A]_{p}$ and call it the Lie derivative of $A$ with respect to $L$ at $p$. If $L[A]_{p}$ exists at all $p \in \mathcal{X}$ we denote by $L[A]$ the mapping $p \rightarrow L[A]_{p}$.

The derivative above is the ordinary weak derivative. Thus to show that $L[A]_{p}$ exists we must show that whenever $l$ is an element of the space conjugate to the space of tensors at $p$ of the same variance as $A_{p}$, then $(d / d t)_{t=0} l\left(\delta \phi_{-t}\left(A_{\phi_{t}(p)}\right)\right)$ exists. Since $l \rightarrow(d / d t)_{t=0} l\left(\delta \phi_{-t}\left(A_{\phi_{t}(p)}\right)\right)$ will then obviously be a linear map, we can by reflexivity identify it with a unique tensor $L[A]_{p}$ at $p$ of the same variance as $A_{p}$. We now show that if $A$ is a $C^{\infty}$ tensor field, then $L[A]_{p}$ always exists and that $L[A]$ is a $C^{\infty}$ tensor field.

Lemma a. If $f$ is a $C^{\infty}$ function on $\mathcal{X}$, then $L[f]_{p}$ exists for each $p \in \mathcal{X}$ and equals $L_{p} f$.

PROOF. $\delta \phi_{-t}\left(f_{\phi_{t}(p)}\right)=f(\phi(t, p))$ so $(d / d t)_{t=0}\left(\delta \phi_{-t}\left(f_{\phi_{t}(p)}\right)\right)=(d / d t)_{t=0}$ $f(\phi(t, p))=L_{p} f$. q.e.d.

Lemma b. If $f$ is a $C^{\infty}$ function on $\mathcal{X}$, then $L[d f]_{p}$ exists for each $p \in \mathcal{X}$ and equals $d(L f)_{p}$.

Proof. Let $M$ be a tangent vector at $p$. We must show that $h(t)$ $=\delta \phi_{-t}\left(d f_{\phi_{t}(p)}\right) M=\delta \phi_{t}(M) f=M\left(f \circ \phi_{t}\right)$ is differentiable at $t=0$ and has derivative $d(L f)_{p}(M)$ there. Now since $\phi$ is a $C^{\infty}$ mapping, $f \circ \phi$ is of class $C^{\infty}$ in a neighborhood of $(0, p) \in R \times \mathcal{H}$. Since $(d / d y)_{y=0}$ and $M$ are tangent vectors at this point in different factor spaces, they commute and we have

$$
\begin{aligned}
h^{\prime}(0) & =(d / d t)_{t=0} M\left(f \circ \phi_{t}\right)=M\left((d / d t)_{t=0} f \circ \phi_{t}\right) \\
& =M(L f)=d(L f)_{p}(M) .
\end{aligned}
$$


Lemma c. If $M$ is a $C^{\infty}$ vector field on $\mathscr{H}$ and $p \in \mathcal{H}$, then $L[M]_{p}$ exists and equals $[L, M]_{p}$.

PROOF. The most general linear functional on the tangent space at $p$ is of the form $d f_{p}$ where $f$ is function of class $C^{\infty}$ at $p$. Thus we must show that if $f$ is a $C^{\infty}$ function on $\mathcal{X}$ and $h(t)=d f\left(\delta \phi_{-t}\left(M_{\phi_{t}(p)}\right)\right)$ $=\delta \phi_{-t}\left(M_{\phi_{t}(p)}\right) f=M_{\phi_{t}(p)}\left(f \circ \phi_{-t}\right)$, then $h$ is differentiable at zero and $h^{\prime}(0)=d f\left([L, M]_{p}\right) \equiv[L, M]_{p} f \equiv L_{p}(M f)-M_{p}(L f)$. Writing $F(x, y)$ $=M_{\phi_{x}(p)}\left(f \circ \phi_{-y}\right)$ we have $h^{\prime}(0)=F_{1}(0,0)+F_{2}(0,0)$. Now $F_{1}(0,0)$ $=(d / d x)_{x=0}\left(M_{\phi_{x}(p)} f\right)=(d / d x)_{x=0}((M f)(\phi(x, p)))=L_{p}(M f)$ and by reasoning similar to that in Lemma $\mathrm{b}, F_{2}(0,0)=(d / d y)_{y=0}\left(M_{p}\left(f \circ \phi_{-y}\right)\right)$ $=-(d / d y)_{y=0}\left(M_{p}\left(f \circ \phi_{y}\right)\right)=-M_{p}(L f)$. q.e.d.

Lemma d. Let $A$ and $B$ be two tensor fields on $\mathcal{X}$ such that $L[A]_{p}$ and $L[B]_{p}$ exist. Then $L[A \times B]_{p}$ exists and equals $L[A]_{p} \otimes B_{p}+A_{p} \otimes L[B]_{p}$ and if $A$ and $B$ have the same variance, then $L[A+B]_{p}$ exists and equals $L[A]_{p}+L[B]_{p}$.

Proof. The second statement is obvious. As for the first we have $\delta \phi_{-t}(A \otimes B)_{\phi_{t}(p)}=\delta \phi_{-t}(A)_{\phi_{t}(p)} \otimes \delta \phi_{-t}(B)_{\phi_{t}(p)}$. Thus if $l_{1}$ is a linear functional on the space of tensors at $p$ of the same variance as $A_{p}$, and $l_{2}$ a linear functional on the space of tensors at $p$ of the same variance as $B_{p}$, then

$$
\begin{aligned}
(d / d t)_{t=0} l_{1} \otimes l_{2}\left(\delta \phi_{-t}(A \otimes B)_{\phi_{t}(p)}\right) & \\
& =(d / d t)_{t=0}\left[l_{1}\left(\delta \phi_{-t}\left(A_{\phi_{t}(p)}\right)\right) l_{2}\left(\delta \phi_{-t}\left(B_{\phi_{t}(p)}\right)\right)\right] \\
& =l_{1}\left(L[A]_{p}\right) l_{2}\left(B_{p}\right)+l_{1}\left(A_{p}\right) l_{2}\left(L[B]_{p}\right) \\
& =l_{1} \otimes l_{2}\left(L[A]_{p} \otimes B_{p}+A_{p} \otimes L[B]_{p}\right) .
\end{aligned}
$$

Since the linear functionals on the space of tensors at $p$ of the same variance as $(A \otimes B)_{p}$ which are of the form $l_{1} \otimes l_{2}$ span, this proves the lemma. q.e.d.

Clearly if two tensor fields agree in the neighborhood of a point $p$ and the Lie derivative of one exists at $p$, then the Lie derivative of the other exists there also and is equal to that of the first. Since in a neighborhood of any point an arbitrary $C^{\infty}$ tensor field is equal to the sum of tensor products of $C^{\infty}$ functions, the differentials of $C^{\infty}$ functions, and $C^{\infty}$ vector fields, we get the following consequence of Lemmas a through $\mathrm{d}$.

Theorem II. If $A$ is a $C^{\infty}$ tensor field on $\mathcal{X}$, then $L[A]_{p}$ exists for every $p \in \mathcal{X}$ and $L[A]$ is a $C^{\infty}$ tensor field of the same variance as $A$.

Theorem III. If $L_{1}$ and $L_{2}$ are two $C^{\infty}$ vector fields on $\mathcal{X}$ and $A$ is a $C^{\infty}$ tensor field on $\mathcal{X}$, then $\left(L_{1}+L_{2}\right)[A]=L_{1}[A]+L_{2}[A]$. 
Proof. By Lemmas a, b, and c the theorem holds if $A$ is a $C^{\infty}$ function, the differential of a $C^{\infty}$ function, or a $C^{\infty}$ vector field. By Lemma $d$, if it holds for two tensor fields it holds for their outer product, and if they are of the same variance, for their sum. The reasoning for Theorem II shows that it holds in general. q.e.d.

If $\psi$ is a nonsingular $C^{\infty}$ map of $\mathcal{X}$ into $\mathscr{X}$ defined near $p$, then it is clear that the isomorphisms $\delta \psi$ of the tensor spaces at $p$ with those at $\psi(p)$ commute with all contraction operators. Thus follows:

Theorem IV. The Lie derivative commutes with contractions.

Theorem V. Let $A$ be a $C^{\infty}$ tensor field of contravariant rank $r$ and covariant rank s. Let $\Theta_{1}, \cdots, \Theta_{r}$ be $r$ covariant vector fields and $M_{1}, \cdots, M_{8}$ be sontravariant vector fields. Then

$$
\begin{aligned}
L\left[A\left\langle\Theta_{1}, \cdots, \Theta_{r}, M_{1}, \cdots, M_{s}\right\rangle\right]=L[A]\left\langle\Theta_{1}, \cdots, M_{s}\right\rangle \\
+\sum_{i=1}^{r} A\left\langle\Theta_{1}, \cdots, L\left[\Theta_{i}\right], \cdots, \Theta_{r}, M_{1}, \cdots, M_{s}\right\rangle \\
+\sum_{i=1}^{s} A\left\langle\Theta_{1}, \cdots, \Theta_{r}, M_{1}, \cdots, L\left[M_{i}\right], \cdots, M_{s}\right\rangle .
\end{aligned}
$$

Proof. We note that $A\left\langle\Theta_{1}, \cdots, \Theta_{r}, M_{1}, \cdots, M_{s}\right\rangle$ is the complete contraction of $A \otimes \Theta_{1} \otimes \cdots \otimes \Theta_{r} \otimes M_{1} \otimes \cdots \otimes M_{2}$, and use Lemma $\mathrm{d}$ and Theorem IV. q.e.d.

Corollary. If $\Theta$ is a $C^{\infty} k$-form on $\mathcal{X}$ (i.e. an alternating covariant tensor field of rank $k$ ) and $M_{1}, \cdots, M_{k}$ are $k C^{\infty}$ vector fields on $\mathcal{X l}$ then:

$$
\begin{aligned}
& L\left[\Theta\left\langle M_{1}, \cdots, M_{k}\right\rangle\right]=L[\Theta]\left\langle M_{1}, \cdots, M_{k}\right\rangle \\
&+\sum_{i=1}^{k}(-1)^{i+1} \Theta\left\langle\left[L, M_{i}\right], M_{1}, \cdots, \widehat{M}_{i}, \cdots, M_{k}\right\rangle .
\end{aligned}
$$

Proof. An immediate consequence of the theorem, Lemma c, and the alternating character of $\Theta$.

We write successive Lie derivatives of a tensor field $A$ as $L^{2}[A]$, $L^{3}[A]$, etc. We write $A=L^{0}[A]$. The following generalization of Taylor's theorem with remainder seems to be new.

Theorem VI. If $A$ is a $C^{\infty}$ tensor field on $\mathcal{X}, L$ a $C^{\infty}$ vector field on $\mathcal{X}, \phi$ the maximal quasi group it generates, and if $(t, p)$ is in the domain of $\phi$, then

$$
\delta \phi_{-t}\left(A_{\phi_{t}(p)}\right)=\sum_{i=0}^{n}\left(t^{i} / i !\right) L^{i}[A]_{p}+R_{n}
$$

where $R_{n}=\int_{0}^{t}(t-\xi)^{n} / n ! \delta \phi_{-\xi}\left(L^{n+1}[A]_{\phi \xi(p)}\right) d \xi$, the integral being a Pettis 
(i.e. weak) vector-valued integral.

ProOF.

$$
\begin{aligned}
(d / d \tau)\left(\delta \phi_{-\tau} A_{\phi_{\tau}(p)}\right) & =(d / d \xi)_{\xi=0}\left(\delta \phi_{-(\tau+\xi)} A_{\phi_{\tau+\xi}(p)}\right) \\
& =(d / d \xi)_{\xi=0}\left(\delta \phi_{-\tau} \delta \phi_{-\xi} A_{\phi_{\xi} \phi_{\tau}(p)}\right) \\
& =\delta \phi_{-\tau}\left[(d / d \xi)_{\xi=0}\left(\delta \phi_{-\xi} A_{\phi_{\xi} \phi_{\tau}(p)}\right)\right] \\
& =\delta \phi_{-\tau}\left(L[A]_{\phi_{\tau}(p)}\right) .
\end{aligned}
$$

Integrating from $\tau=0$ to $\tau=t$ and setting $w=\xi_{1}, x=\xi_{2}, y=\xi_{n}, z=\xi_{n+1}$, we get $\delta \phi_{-t}\left(A_{\phi_{t}(p)}\right)=A_{p}+\int_{0}^{t} \delta \phi_{-v}\left(L[A]_{\phi_{w}(p)}\right) d w$. Replacing $A$ by $L[A]$ and substituting back, we get in $n$ steps

$$
\begin{aligned}
\delta \phi_{-t}\left(A_{\phi_{t}(p)}\right)= & \sum_{i=0}^{n}\left(t^{i} / i !\right) L^{i}[A]_{p} \\
& +\int_{0}^{t} d w \int_{0}^{w} d x \cdots d y \int_{0}^{y} d z \delta \phi_{-z}\left(L[A]_{\phi_{z}(p)}\right),
\end{aligned}
$$

and the well known formula

$$
\int_{0}^{t} d \xi_{1} \int_{0}^{\xi_{1}} d \xi_{2} \cdots \int_{0}^{\xi_{n}} d \xi_{n+1} f\left(\xi_{n+1}\right)=\int_{0}^{t}(t-\xi)^{n} / n ! f(\xi) d \xi
$$

completes the proof. q.e.d.

Corollary. $L[A]=0$ is a necessary and sufficient condition that $\delta \phi_{t}\left(A_{p}\right)=A_{\phi_{t}(p)}$ for all $(t, p)$ in the domain of $\phi$.

The exterior derivative. We are now in a position to give a coordinate free definition of the exterior derivative in terms of Lie derivatives.

Definition. Let $M_{1}, \cdots, M_{k+1}$ be $k+1 C^{\infty}$ vector fields on a $C^{\infty}$ manifold $\mathcal{H}$. We define an operator $d_{M_{1}, \cdots, M_{k+1}}$ mapping $C^{\infty} k$-forms $\Theta$ on $\mathscr{X}$ into $C^{\infty}$ functions on $\mathcal{X}$ by

$$
\begin{aligned}
d_{M_{1}}, \cdots, M_{k+1} \Theta=\frac{1}{2} \sum_{i=1}^{k+1}(-1)^{i+1}\{ & M_{i}\left[\Theta\left\langle M_{1}, \cdots, \widehat{M}_{i}, \cdots, M_{k+1}\right\rangle\right] \\
& \left.+M_{i}[\Theta]\left\langle M_{1}, \cdots, \widehat{M}_{i}, \cdots, M_{k+1}\right\rangle\right\} .
\end{aligned}
$$

We note that from the definition it is obvious that $M_{1}, \cdots, M_{k+1}$ $\rightarrow d_{M_{1}, \cdots, M_{k+1}}$ is an alternating multilinear mapping, also that if $\mathcal{O}$ is an open subset of $\mathcal{X}, \Theta=\Theta^{\prime}$ in $\mathcal{O}$ and $M_{i}=M_{i}^{\prime}$ in $\mathcal{O}, i=1,2, \cdots$, $k+1$, then $d_{M_{1}, \cdots, M_{k+1}} \Theta=d_{M_{1}^{\prime}}^{\prime}, \cdots, M_{k+1}^{\prime} \Theta^{\prime}$ in $\mathcal{O}$.

From the corollary of Theorem $\mathrm{V}$ we get the following two lemmas by a trivial calculation. 
LEMma 1.

$$
\begin{aligned}
& d_{M_{1}, \cdots, M_{k+1}} \Theta=\sum_{i=1}^{k+1}(-1)^{i+1} M_{i}\left[\Theta\left\langle M_{1}, \cdots, \widehat{M}_{i}, \cdots, M_{k+1}\right\rangle\right] \\
& +\sum_{1 \leqq i<j \leqq k+1}(-1)^{i+i} \Theta\left\langle\left[M_{i}, M_{j}\right], M_{1}, \cdots, \widehat{M}_{i}, \cdots, \widehat{M}_{j}, \cdots, M_{k+1}\right\rangle .
\end{aligned}
$$

LEMMA 2.

$$
\begin{aligned}
& d_{M_{1}, \cdots, M_{k+1}} \Theta=\sum_{i=1}^{k+1}(-1)^{i+1} M_{i}[\Theta]\left\langle M_{1}, \cdots, \widehat{M}_{i}, \cdots, M_{k+1}\right\rangle \\
& +\sum_{1 \leqq i<j \leqq k+1}(-1)^{i+j+1} \Theta\left\langle\left[M_{i}, M_{j}\right], M_{1}, \cdots, \widehat{M}_{i}, \cdots, \widehat{M}_{j}, \cdots, M_{k+1}\right\rangle .
\end{aligned}
$$

The right-hand side of Lemma 1 is a known formula for $(d \Theta)$ $\left\langle M_{1}, \cdots, M_{k+1}\right\rangle$. The usual procedure is to define the exterior derivative by using coordinates, prove it is a differential form, and then prove Lemma 1. A direct proof that the right-hand side of Lemma 1 is a differential form is difficult. The advantage of the present approach is that, as we shall see below, we can prove easily and without using coordinates that the right-hand side of Lemma 2 (which uses the concept of Lie derivative explicitly) defines a differential form.

Theorem VII. Let $\Theta$ be a $C^{\infty} k$-form on a $C^{\infty}$ manifold $\mathcal{X}$. There exists a unique differential $k+1-$ form $\Phi$ on $\mathcal{X}$ such that for any $k+1$ $C^{\infty}$ vector fields $M_{1}, \cdots, M_{k+1}$ on $\mathcal{X}$ we have

$$
\Phi\left\langle M_{1}, \cdots, M_{k+1}\right\rangle=d_{M_{1}}, \cdots, M_{k+1} \Theta .
$$

Proof. Let $p \in \mathcal{X}$ and let $M_{1}^{(p)}, \cdots, M_{k+1}^{(p)}$ be $k+1$ tangent vectors at $p$. Then as is well known and obvious we can find $k+1 C^{\infty}$ vector fields on $\mathcal{X}, M_{1}, \cdots, M_{k+1}$, such that $\left(M_{i}\right)_{p}=M_{i}^{(p)}$. Then if $\Phi$ does in fact exist we must have

$$
\Phi_{p}\left\langle M_{1}^{(p)}, \cdots, M_{k+1}^{(p)}\right\rangle=\left(d_{M_{1}, \cdots, M_{k+1}} \Theta\right)(p) .
$$

To prove that the function $\Phi$ defined on the cartesian product of the tangent space at $p$ with itself $k+1$ times by the above formula is well defined, we must show that it is independent of the vector field $M_{i}$ chosen to extend the $M_{i}^{(p)}$. Because of the alternating multilinear character of the right-hand side it is sufficient to show that if $M_{1}, \cdots, M_{k+1}$ are $k+1 C^{\infty}$ vector fields such that $M_{1}$ vanishes at $p$, then $\left(d_{M_{1}}, \ldots, M_{k+1} \Theta\right)(p)=0$. Now remembering that if $\Pi$ is a $k$-form on $\mathcal{X}$ and $L_{1}, \cdots, L_{k}$ are $k$ vector fields on $\mathcal{X}$, then $\Pi\left\langle L_{1}, \cdots, L_{k}\right\rangle$ vanishes at any point where one of the $L_{i}$ vanish, we have from Lemma 2 under the assumption $\left(M_{1}\right)_{p}=0$ that 


$$
\begin{aligned}
& \left(d_{M_{1}}, \cdots, M_{k+1} \Theta\right)(p)=M_{1}[\Theta]_{p}\left\langle M_{2}, \cdots, M_{k+1}\right\rangle \\
& +\sum_{j=2}^{k+1}(-1)^{j} \Theta_{p}\left\langle\left[M_{1}, M_{j}\right], M_{2}, \cdots, \widehat{M}_{i}, \cdots, M_{k+1}\right\rangle
\end{aligned}
$$

which by the corollary of Theorem $\mathrm{V}$

$$
=M_{1}\left[\Theta\left\langle M_{2}, \cdots, M_{k+1}\right\rangle\right]_{p}
$$

and this by Lemma a of Theorem II

$$
=\left(M_{1}\right)_{p} \Theta\left\langle M_{2}, \cdots, M_{k+1}\right\rangle
$$

which vanishes since by hypothesis $\left(M_{1}\right)_{p}=0$. Thus $\Phi_{p}$ is a well defined function. It is alternating and multilinear since $M_{1}, \cdots, M_{k+1}$ $\rightarrow d_{M_{1}, \cdots, M_{k+1}}$ is, and so is a differential $k+1$ form at $p$. Finally $\Phi: p \rightarrow \Phi_{p}$ is of class $C^{\infty}$ since if $M_{1}, \cdots, M_{k+1}$ are $k+1 C^{\infty}$ vector fields on $\mathcal{X}$, then $\Phi\left\langle M_{1}, \cdots, M_{k+1}\right\rangle=d_{M_{1}, \cdots, M_{k+1}} \Theta$ and the latter is a $C^{\infty}$ function on $\mathcal{X}$. This completes the proof of the theorem. q.e.d.

Definition. The differential $k+1$ form $\Phi$ defined above is called the exterior derivative of $\Theta$ and is denoted by $d \Theta$.

We have still to show that what we have defined is the usual exterior derivative. Since $\Theta \rightarrow d \Theta$ as defined above is clearly linear it will suffice to show that if $f$ is a $C^{\infty}$ function on $\mathcal{X}, x_{1}, \cdots, x_{n}$ a coordinate system, and $\Theta=f d x_{1} \wedge \cdots \wedge d x_{k}$ in the domain of $x_{1}, \cdots, x_{k}$, then $d \Theta=d f \wedge d x_{1} \wedge \cdots \wedge d x_{k}$ in the domain of $x_{1}, \cdots, x_{n}$. Now using the fact that $d x_{i}\left\langle\partial / \partial x_{j}\right\rangle=\delta_{i j}$ and $\left[\partial / \partial x_{i}, \partial / \partial x_{j}\right]=0$ it is easy to see from Lemma 1 that $d \Theta\left\langle\partial / \partial x_{i_{1}}, \cdots, \partial / \partial x_{i_{k+1}}\right\rangle=0$ unless with the omission of an $i,\left(i_{1}, \cdots, i_{k+1}\right)$ is a permutation of $(1,2, \cdots, k)$ and the omitted $i$ is greater than $k$, and that for $j>k, d \Theta\left\langle\partial / \partial x_{1}, \cdots\right.$, $\left.\partial / \partial x_{k}, \partial / \partial x_{j}\right\rangle=(-1)^{j} \partial f / \partial x_{j}$. Hence

$$
\begin{aligned}
d \Theta & =\sum_{i_{1}<\cdots<i_{k+1}} d \Theta\left\langle\partial / \partial x_{i_{1}}, \cdots, \partial / \partial x_{i_{k+1}}\right\rangle d x_{i_{1}} \wedge \cdots \wedge d x_{i_{k+1}} \\
& =\sum_{j>k} d \Theta\left\langle\partial / \partial x_{1}, \cdots, \partial / \partial x_{k}, \partial / \partial x_{j}\right\rangle d x_{1} \wedge \cdots \wedge d x_{k} \wedge d x_{j} \\
& =\sum_{j=1}^{n} \partial f / \partial x_{j} d x_{j} \wedge d x_{1} \wedge \cdots \wedge d x_{k}=d f \wedge d x_{1} \wedge \cdots \wedge d x_{k} . \quad \text { q.e.d. }
\end{aligned}
$$

\section{REFERENCES}

1. J. A. Schouten and W. v. d. Kulk, Pfaff's problem and its generalizations, Oxford, 1949.

2. S. S. Chern, Notes on differentiable manifolds, Mimeographed at University of Chicago.

HARVARD UNIVERSITY 